\title{
Transverse single spin asymmetry in direct photon production in polarized pA collisions
}

\author{
Andreas Schäfer and Jian Zhou \\ Institut für Theoretische Physik,Universität Regensburg, Regensburg, Germany
}

July 13, 2021

\begin{abstract}
We study the transverse single spin asymmetry in direct photon production in pA collisions with incoming protons being transversely polarized. To facilitate the calculation, we formulate a hybrid approach in which the nucleus is treated in the Color Glass Condensate (CGC) framework while the collinear twist-3 formalism is applied on the proton side. It has been found that an additional term which arises from color entanglement shows up in the spin dependent differential cross section. The fact that this additional term is perturbatively calculable allows us to quantitatively study color entanglement effects.
\end{abstract}

\section{Introduction}

The phenomenology of transverse single spin asymmetries (SSAs) in high energy scattering has attracted a lot of attention and has been under intense investigation during the past few decades. The large size of the observed SSAs for single inclusive hadron production came as a big surprise and can not be understood in the naive parton model [1,2]. It signals that QCD phenomena are in general much richer than in the well studied collinear limit. This opens up many possibilities. For example, SSAs could be especially sensitive to saturation phenomena. However, QCD beyond the collinear limit is usually more difficult and less under theoretical control, such that presently one is still searching for the most adequate theoretical frameworks to treat them. This contribution constitutes one more attempt along these lines.

Various recent studies show that it is necessary to take into account initial/final state gluon rescattering interactions in order to generate large SSAs. Two ways of incorporating these initial/final state interactions are based on the transverse momentum dependent(TMD) factorization [3, 4] and the collinear twist-3 factorization [5-10], respectively. In TMD factorization, the naive time reversal odd TMD distributions and fragmentation function, known as the quark/gluon Sivers functions [3] and the Collins fragmentation function [4] can account for the large SSAs, while in the collinear twist3 approach, SSAs can arise from a twist-3 quark gluon correlator, the so-called Efremov-TeryaevQiu-Sterman(ETQS) function [5, 6], a tri-gluon correlation functions [8, 9], and a twist-3 collinear fragmentation functions [10]. It has been established that the $k_{\perp}$ moment of the Sivers function and the Collins function can be related to the ETQS function and the corresponding twist-3 collinear fragmentation functions, respectively [10,11].

SSAs observed in various processes like pion production in single polarized pp collisions $p^{\uparrow} p \rightarrow \pi X$ or in SIDIS $e p^{\uparrow} \rightarrow \pi X$ receive contributions from both sources: the Sivers mechanism and the Collins mechanism. A very recent study has shown that the Collins effect described within the collinear twist3 framework might be the dominant contribution to the spin asymmetry in polarized pp collisions [12]. 
Nevertheless, it would be crucial to unambiguously pin down the Sivers mechanism in polarized pp collisions, as the so-called "sign mismatch" problem [13] (for a different version of this problem, see also [14]) is still unsolved. It is thus desirable to investigate SSAs for cases of particle production in polarized $p p$ collisions for which the Collins effect is absent. Possible options are the SSA in direct photon production [7, 15, 18], jet production [16 18], or heavy quarkonium production in polarized pp collisions [19,20]. The SSA for direct photon production in polarized pp collisions has been calculated in the collinear twist-3 approach in [7, 15, 18]. In the present work, we extend this analysis to single polarized $\mathrm{p}^{\uparrow} \mathrm{A}$ collisions.

Though most work in this field focuses on SSAs in $\mathrm{ep}^{\uparrow}$ or $\mathrm{pp}^{\uparrow}$ collisions, there exist a few exploratory investigations devoted to the study of SSAs in $\mathrm{p}^{\uparrow} \mathrm{A}$ collisions. The authors of Ref. [21 investigated the SSA for inclusive pion production at forward rapidities in $\mathrm{p}^{\uparrow} \mathrm{p}$ collisions using a hybrid approach in which the target proton is treated in the CGC framework [22] while the spin-transverse momentum correlation in the projectile proton is described by the Sivers distribution. Their analysis can be straightforwardly applied to $\mathrm{p}^{\uparrow} \mathrm{A}$ collisions. Following the same line of reasoning, the SSA of DrellYan lepton pairs produced in $\mathrm{p}^{\uparrow} \mathrm{A}$ collisions was computed in [23]. On the other hand, the SSA for inclusive pion production caused by the Collins mechanism after the transversely polarized quark from the projectiles is scattered off the background gluon field of the nucleus was investigated in Ref. 24]. Furthermore, a recent GCG calculation suggests that SSAs also can be generated by the interaction of the spin-dependent light-cone wave function of the projectile with the target gluon field via C-odd odderon exchange [25].

The purpose of the present work is to study SSA for direct photon production in $\mathrm{p}^{\uparrow} \mathrm{A}$ collisions and to decide whether it provides a sensitive tool to establish and study saturation effects. First one can note that the contribution from fragmentation to the spin asymmetry for direct photon production is found to be negligible [17]. Next one observes that TMD factorization can not be applied on proton side for lack of an additional hard scale. Moreover, it has been shown that the odderon exchange does not give rise to a SSA for direct photon production [25]. Therefore, the only possible source for a sizeable SSA is the Sivers effect which can only be described within the collinear twist-3 approach for the process under consideration. To do so, we formulate a novel hybrid approach in which the nucleus is treated in the Color Glass Condensate (CGC) framework while the collinear twist-3 formalism is applied on the proton side. In this hybrid approach, we take into account one extra gluon exchange from the proton side and sum gluon re-scattering to all orders on the nucleus side.

The resulting spin dependent differential cross section computed in this hybrid approach is proportional to a convolution of the ETQS function and various Wilson lines. These Wilson lines can be further related to two different types of $k_{\perp}$ dependent gluon distributions, one of which is the dipole type gluon TMD. The other arises from a color entanglement effect which is due to the non-trivial interplay of gluons from both the nucleon and nucleus [26]. This effect seems to be a unique feature of non-abelian theories, i.e. it is linked to one of the most fundamental aspects of QCD. The fact that this additional term can be perturbatively calculated in the Mclerran-Venugopalan (MV) model [22] allows us to quantitatively study the color entanglement effect and to test the MV model. A measurement of this observable would also provide a hint to the size of generalized TMD factorization breaking effect. Let us note that it was argued that such a color entanglement effect could also manifest itself through azimuthal asymmetries in the Drell-Yan process [27].

In a more general context, the present work is part of the effort to address the interplay between spin physics and saturation physics. Apart from the studies mentioned above, early work in this very active field includes the study of small $\mathrm{x}$ evolution of spin dependent structure function $g_{1}$ [28] and of the quark Boer-Mulders distribution and the linearly polarized gluon distribution inside a large nucleus, see Refs. [29]. The small x evolution equations for the linearly polarized gluon distributions were derived in Ref. [30]. Several ways of accessing the linearly polarized gluon distributions inside 
a large nucleus have been discussed in 29 32. Furthermore, the asymptotic behavior of transverse single spin asymmetries at small $\mathrm{x}$ was discussed in Ref. [33, 34. It has been shown that SSAs at small $\mathrm{x}$ are generated by polarized odderon exchange whose size is determined by the anomalous magnetic moment [34. The quark Sivers function was computed in the quasi-classical GlauberMueller/MV approximation [35]. More recently, the authors of the paper [36] have investigated the spin asymmetries in pA collisions by going beyond the Eikonal approximation.

The paper is organized as follows. In section II, we briefly review the existing calculations for direct photon production, including the collinear twist-3 calculation for direct photon production in polarized $\mathrm{p}^{\uparrow} \mathrm{p}$ collisions and the CGC calculation for direct photon production in unpolarized pA collisions. In section III, we develop the hybrid approach and explain all technical steps in details. We focus on the derivative term contribution and identify a term arising from the color entanglement effect. It is shown that the spin dependent differential cross section derived in collinear factorization can be recovered from our result without color entanglement effect being incorporated in the kinematical limit where the produced photon transverse momentum is much larger than the saturation scale. The paper is summarized in section IV.

\section{Brief review of existed calculations for direct photon production}

In this section, we review how the calculation of the SSA for direct photon production is formulated within the collinear twist- 3 approach in $\mathrm{p}^{\uparrow} \mathrm{p}$ collisions, following by a brief reminder of the application of the CGC framework to direct photon production in unpolarized pA collisions.

\subsection{SSA in direct photon production in $\mathrm{p}^{\uparrow} \mathrm{p}$ collisions}

The dominant production mechanism for prompt photons in high energy collisions is Compton scattering $g q \rightarrow \gamma q$. We start by introducing the relevant kinematical variables and assign 4-momenta to the particles according to

$$
g\left(x_{g}^{\prime} \bar{P}\right)+q(x P) \longrightarrow \gamma\left(l_{\gamma}\right)+q\left(l_{q}\right)
$$

where $\bar{P}^{\mu}=\bar{P}^{-} n^{\mu}$ and $P^{\mu}=P^{+} p^{\mu}$ with $n^{\mu}$ and $p^{\mu}$ being the commonly defined light cone vectors, normalized according to $p \cdot n=1$. The Mandelstam variables are defined as: $S=(P+\bar{P})^{2}, T=\left(P-l_{q}\right)^{2}$ and $U=\left(P-l_{\gamma}\right)^{2}$. The corresponding unpolarized Born cross section reads,

$$
\frac{d^{3} \sigma}{d^{2} l_{\gamma \perp} d z}=\frac{\alpha_{s} \alpha_{e m}}{N_{c}} \frac{z\left[1+(1-z)^{2}\right]}{l_{\gamma \perp}^{4}} \sum_{q} e_{q}^{2} \int_{x_{m i n}}^{1} d x f_{q}(x) x_{g}^{\prime} G\left(x_{g}^{\prime}\right)
$$

where $z \equiv l_{\gamma} \cdot n /(x P \cdot n)$ is the fraction of the incoming quark momentum $x P$ carried by the outgoing photon, and $l_{\gamma \perp}$ is the photon transverse momentum. The meaning of the other coefficients should be self-evident. Note that $x_{g}^{\prime}=\frac{-x T}{x S+U}$ is a function of $x$; and $x_{\min }$ is given by $x_{\min }=\frac{-U}{S+T}$. In the above formula, $f_{q}(x)$ and $G\left(x_{g}^{\prime}\right)$ are the usual integrated quark and gluon distributions, respectively.

To generate the spin asymmetry, one additional gluon must be exchanged between the active partons and the remanent part of the polarized proton projectile. The hard part, if an additional gluon is attached, can be calculated perturbatively, while the non-perturbative part describes the relevant three parton correlations. The strong interaction phase factor necessary for having a non-vanishing spin asymmetry arises from the interference between an imaginary part of the partonic scattering amplitude with an extra gluon and the real scattering amplitude without a gluon attachment, as shown in Fig.1. The imaginary part is due to the pole of the parton propagator associated with the integration over the gluon momentum fraction $x_{g}$. This effectively implies that one of the internal 


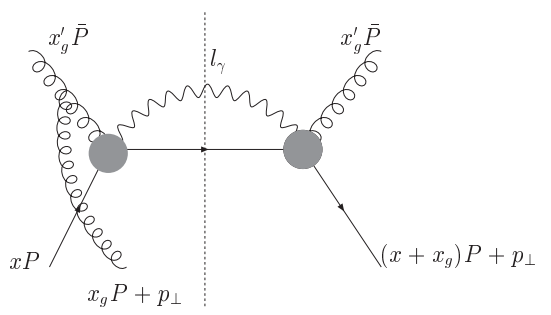

(a)

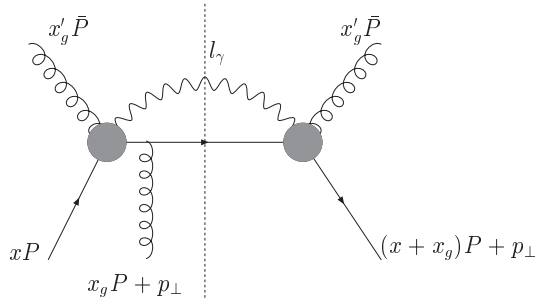

(c)

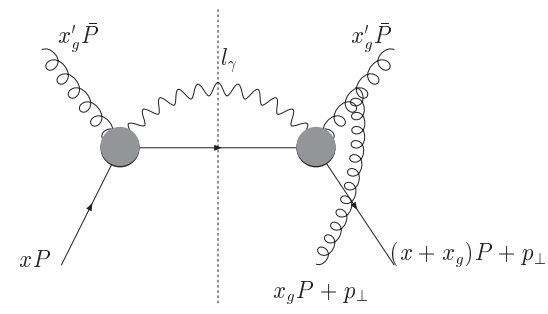

(b)

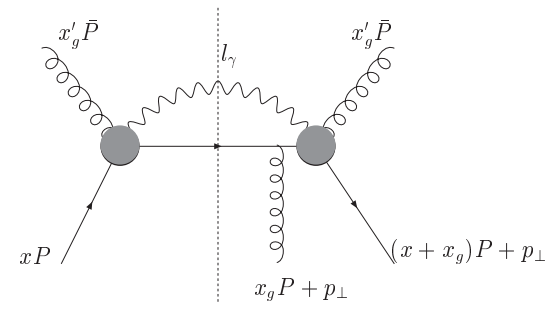

$(d)$

Figure 1: Diagrams contributing to the single spin asymmetry for direct photon production in $\mathrm{p}^{\uparrow} \mathrm{p}$ collisions. Grey circles indicate all possible photon line attachments. The mirror diagrams are not shown here. The contributions from diagrams (c) and (d) to the spin asymmetry cancel.

parton lines goes on shell. To isolate the imaginary part of such poles, the identity of distributions: $\frac{1}{x_{g} \pm i \epsilon}=\mathrm{PV} \frac{1}{x_{g}} \mp i \pi \delta\left(x_{g}\right)$ was used. Depending on which propagator's pole contributes, the amplitude may get contributions from $x_{g}=0$ ("soft-pole") and $x_{g} \neq 0$ ("hard-pole").

It is convenient to carry out the calculation in the covariant gauge, in which the leading contribution of the exchanged gluon is the "plus" component $A^{+}$. The gluon's momentum is given by $p_{g}=x_{g} P+p_{\perp}$, where $x_{g}$ is the longitudinal momentum fraction with respect to the polarized proton. In order to calculate consistently with twist-3 accuracy, one has to expand the hard part in the gluon transverse momentum,

$$
H\left(x_{g} P+p_{\perp}, l_{\gamma}\right)=H\left(x_{g} P, l_{\gamma}\right)+\left.\frac{\partial H\left(x_{g} P+p_{\perp}, l_{\gamma}\right)}{\partial p_{\perp}^{\rho}}\right|_{p_{\perp}=0} p_{\perp}^{\rho}+\ldots
$$

In the above formula, the first term only contributes to the unpolarized Born cross section. We thus have to keep the linear term in $p_{\perp}$ at twist-3 level. In the second term, the $p_{\perp}$ factor can be combined with $A^{+}$to yield $\partial^{\perp} A^{+}$, which is an element of the field strength tensor $F^{\partial+}$. The above expansion allows us to integrate over three of the four components of each of the loop momenta $p_{g}$. The fourdimensional integral is reduced to a convolution in the light-cone momentum fractions of the initial partons. At this step, the relevant three parton correlation can be cast into the form of the ETQS function defined as $[5,6]$,

$$
\begin{aligned}
T_{F, q}\left(x_{1}, x_{2}\right)= & \int \frac{d y_{1}^{-} d y_{2}^{-}}{4 \pi} e^{i x_{1} P^{+} y_{1}^{-}+i\left(x_{2}-x_{1}\right) P^{+} y_{2}^{-}} \\
& \times\left\langle P, S_{\perp}\left|\bar{\psi}_{q}(0) \gamma^{+} g \epsilon^{S_{\perp} \sigma n p} F_{\sigma}^{+}\left(y_{2}^{-}\right) \psi_{q}\left(y_{1}^{-}\right)\right| P, S_{\perp}\right\rangle
\end{aligned}
$$

where we have suppressed Wilson lines. $S_{\perp}$ denotes the proton transverse spin vector. Note that our definition of the ETQS functions differs by a factor $g$ from the convention used in Ref. [15]. This ETQS function plays an important role in describing SSA phenomenology. 


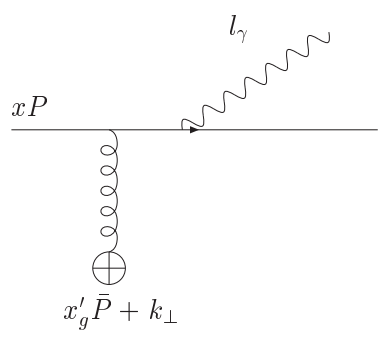

$(a)$

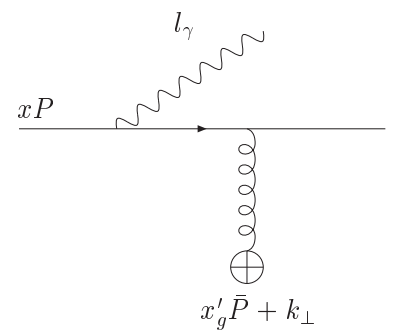

(b)

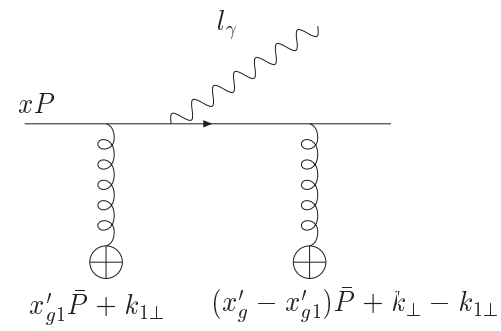

$(c)$

Figure 2: Diagrams contributing to the unpolarized cross section for direct photon production in $\mathrm{pA}$ collisions. The gluon line terminated by a cross surrounded with a circle denotes a classical field $A_{A}$ insertion. The contribution from diagram(c) vanishes because two poles are lying on the same half plane.

Making use of the ingredients described above, the calculation is straightforward. The spin dependent cross section has been calculated and given in [7, 15],

$$
\begin{aligned}
\frac{d^{3} \Delta \sigma}{d^{2} l_{\gamma \perp} d z}= & \frac{\alpha_{s} \alpha_{e m} N_{c}}{N_{c}^{2}-1} \frac{z\left[1+(1-z)^{2}\right]}{l_{\gamma \perp}^{4}}(z-1)\left(\frac{\epsilon^{l_{\gamma} S_{\perp} n p}}{l_{\gamma \perp}^{2}}\right) \\
& \times \sum_{q} e_{q}^{2} \int_{x_{\text {min }}}^{1} d x x_{g}^{\prime} G\left(x_{g}^{\prime}\right)\left[T_{F, q}(x, x)-x\left(\frac{d}{d x} T_{F, q}(x, x)\right)\right]
\end{aligned}
$$

where we have omitted the soft fermion pole contribution [18]. In the next section, we will show that the above spin dependent cross section can be recovered from the proposed hybrid approach in the kinematical limit where the saturation scale is much smaller than the produced photon transverse momentum after neglecting terms arising from color entanglement effect.

\subsection{Photon production in unpolarized pA collisions}

We now move on to review the CGC calculation for direct photon production in unpolarized pA collisions which has been done in Ref. [37]. Roughly speaking, the CGC calculation for this process differs from the collinear factorization calculation in two ways. Firstly, in the small x region, transverse momenta carried by gluons are not necessarily much smaller than their longitudinal momenta. One thus should keep gluon transverse momenta when computing the hard part. We fix kinematical variables accordingly,

$$
g\left(x_{g}^{\prime} \bar{P}+k_{\perp}\right)+q(x P) \longrightarrow \gamma\left(l_{\gamma}\right)+q\left(l_{q}\right) .
$$

Secondly, due to the high gluon number density at small $x$, it is necessary to resum gluon re-scattering to all orders.

The multiple scattering between quark and the classical color field of the nucleus can be readily resummed to all orders [38,39]. This gives rise to a path-ordered gauge factor along the straight line that extends in $x^{+}$from minus infinity to plus infinity. More precisely, for a quark with incoming momentum $l$ and outgoing momentum $l+k$, the path-ordered gauge factor reads,

$$
2 \pi \delta\left(k^{+}\right) n^{\mu}\left[U\left(k_{\perp}\right)-(2 \pi)^{2} \delta\left(k_{\perp}\right)\right],
$$

with

$$
U\left(k_{\perp}\right)=\int d^{2} x_{\perp} e^{i k_{\perp} \cdot x_{\perp}} U\left(x_{\perp}\right),
$$


and

$$
U\left(x_{\perp}\right)=\mathcal{P} e^{i g \int_{-\infty}^{+\infty} d x^{+} A_{A}^{-}\left(x^{+}, x_{\perp}\right) \cdot t},
$$

where $t$ is the generators in the fundamental representation. We use this as building block to compute the amplitude for direct photon production in high energy $p A$ collisions. It is straightforward to obtain the production amplitude for diagram (a) illustrated in Fig. 2 ,

$$
\mathcal{M}_{2 a}=\bar{u}\left(l_{q}\right)(i e) \notin S_{F}\left(x P+x_{g}^{\prime} \bar{P}+k_{\perp}\right) \not h u(x P)\left[U\left(k_{\perp}\right)-(2 \pi)^{2} \delta\left(k_{\perp}\right)\right]
$$

where a delta function is suppressed. In the above formula, $\varepsilon^{\mu}$ is the polarization vector of the produced photon, and $S_{F}\left(x P+x_{g}^{\prime} \bar{P}+k_{\perp}\right)=i \frac{x P+x_{g}^{\prime} \bar{P}+\not \not_{\perp}}{\left(x P+x_{g}^{\prime} P+k_{\perp}\right)^{2}+i \epsilon}$ is the quark propagator. The contribution of diagram (b) in Fig. 2 to the amplitude is similarly given by,

$$
\mathcal{M}_{2 b}=\bar{u}\left(l_{q}\right) \not S_{F}\left(x P-l_{\gamma}\right)(i e) \notin u(x P)\left[U\left(k_{\perp}\right)-(2 \pi)^{2} \delta\left(k_{\perp}\right)\right]
$$

The contribution of diagram (c) in Fig. 2 vanishes. This is so because both $x_{g 1}^{\prime}$ poles lie below the real axis, such that one can close the integration contour above the real axis and get a vanishing contribution. The total amplitude is thus given by $\mathcal{M}_{2}=\mathcal{M}_{2 a}+\mathcal{M}_{2 b}$. By squaring the amplitude, one obtains the cross section [37,

$$
\frac{d \sigma}{d^{2} l_{\gamma \perp} d z}=\frac{\alpha_{e m} \alpha_{s}}{N_{c}} \frac{1}{l_{\gamma \perp}^{2}} \frac{1+(1-z)^{2}}{z} \sum_{q} e_{q}^{2} \int_{x_{m i n}}^{1} d x \int \frac{d^{2} k_{\perp}}{\left(k_{\perp}-l_{\gamma \perp} / z\right)^{2}} x_{g}^{\prime} G_{D P}\left(x_{g}^{\prime}, k_{\perp}\right) f_{q}(x)
$$

where $x_{g}^{\prime} G_{D P}\left(x_{g}^{\prime}, k_{\perp}\right)$ is the dipole type gluon TMD, defined as

$$
x_{g}^{\prime} G_{D P}\left(x_{g}^{\prime}, k_{\perp}\right)=\frac{k_{\perp}^{2} N_{c}}{2 \pi^{2} \alpha_{s}} \int \frac{d^{2} x_{\perp} d^{2} y_{\perp}}{(2 \pi)^{2}} e^{i k_{\perp} \cdot\left(y_{\perp}-x_{\perp}\right)} \frac{1}{N_{c}}\left\langle\operatorname{Tr}\left[U\left(x_{\perp}\right) U^{\dagger}\left(y_{\perp}\right)\right]\right\rangle_{x_{g}^{\prime}}
$$

The Wilson lines appearing in the above formula can be explicitly evaluated in the MV model [22]. The resulting dipole gluon distribution reads

$$
x_{g}^{\prime} G_{D P}\left(x_{g}^{\prime}, k_{\perp}\right)=\frac{k_{\perp}^{2} N_{c}}{2 \pi^{2} \alpha_{s}} \pi R_{0}^{2} \int \frac{d^{2} r_{\perp}}{(2 \pi)^{2}} e^{i k_{\perp} \cdot r_{\perp}} e^{-\frac{1}{4} r_{\perp}^{2} Q_{s q}^{2}}
$$

where $Q_{s q}^{2}=\alpha_{s} C_{F} \mu \ln \frac{1}{r_{\perp}^{2} \Lambda_{Q C D}^{2}}$ is the quark saturation momentum with $\mu$ being the transverse color source density for a nucleus. Here $R_{0}$ is the radius of nucleus.

In the large transverse momentum region $l_{\gamma \perp}^{2} \gg Q_{s q}^{2} \sim k_{\perp}^{2}$, the denominator in Eq. (12) can be approximated as: $1 /\left(k_{\perp}-l_{\gamma \perp} / z\right)^{2} \approx z^{2} / l_{\gamma \perp}^{2}$. After making this approximation and using the relation

$$
\int d^{2} k_{\perp} x_{g}^{\prime} G_{D P}\left(x_{g}^{\prime}, k_{\perp}\right)=x_{g}^{\prime} G\left(x_{g}^{\prime}\right),
$$

one is able to reproduce Eq. (2) which was obtained from collinear factorization.

\section{$3 \quad$ SSA for photon production in $\mathbf{p}^{\uparrow} \mathbf{A}$ collisions}

To calculate the SSA for direct photon production in polarized pA collisions, we have to take into account one extra gluon exchange between the active partons and the remanent part of the polarized proton, while gluon re-scattering inside the nucleus must be resummed to all orders. A typical diagram contributing to this process is illustrated in Fig. 3. It is worthwhile to mention that gluons from the 


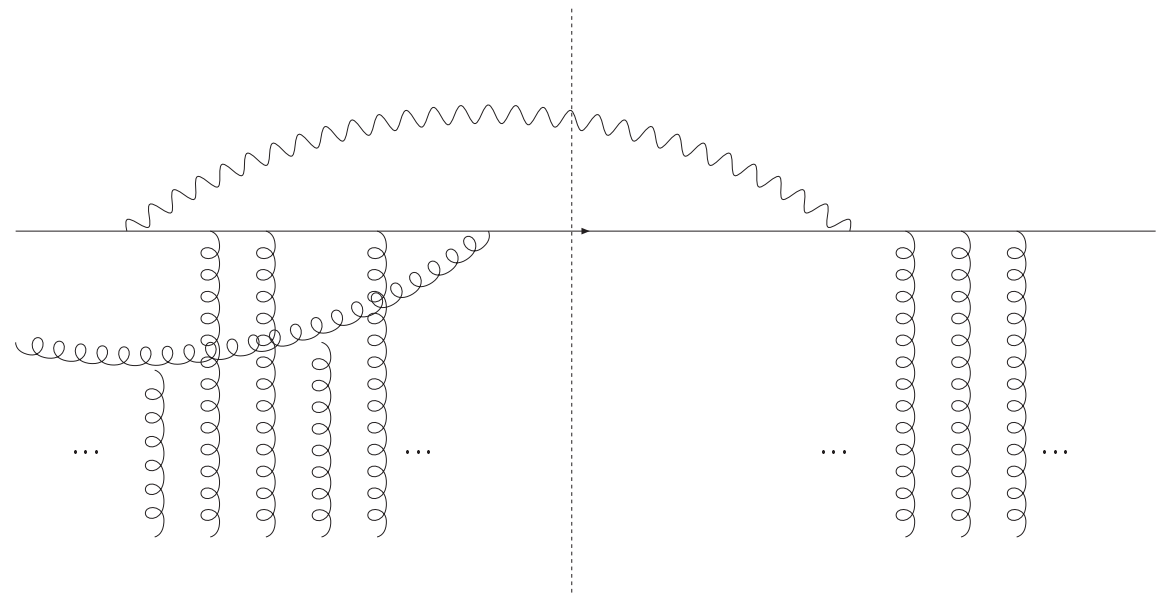

Figure 3: A typical diagram contributing to the SSA in direct photon production in polarized pA collisions. The multiple re-scattering of the incoming partons (including the unpolarized quark and longitudinally polarized gluon) from the proton off the classical gluon field of the nucleus needs to be resummed to all orders.

nucleus could also interact with the color source inside the proton. Such an interaction is not shown in Fig. 3. In this section, we derive the spin dependent amplitude in the CGC framework. We further calculate the derivative term contribution with the obtained amplitude, and also show that the full polarized cross section can be reduced to the one computed from the standard collinear twist-3 approach at high photon transverse momentum provided that the $1 / N_{c}^{2}$ suppressed color entanglement effect has been neglected.

\subsection{Derivation of the spin dependent amplitude}

As mentioned in the previous section, the multiple scattering between the incoming quark and the classical color field of the nucleus can be resummed into a Wilson line. Similarly, this procedure also applies to the case for which the incoming parton is a transversely polarized gluon. However, in the process under consideration, multiple scattering between incoming gluon and background gluon field of the nucleus can not be described by a simple Wilson line, since the incoming gluon from the proton side is longitudinally polarized.

The formula for a longitudinally polarized gluon scattering off a nucleus has been worked out in Ref. [40. The expression for the gauge field created through the fusion of the incoming gluon from the proton and small $\mathrm{x}$ gluons from the nucleus contains both singular terms (proportional to $\delta\left(x^{+}\right)$) and regular terms,

$$
A^{\mu}(q)=A_{r e g}^{\mu}(q)+\delta^{\mu-} A_{\text {sing }}^{-}(q) .
$$

The regular terms $A_{r e g}^{\mu}$ are given by

$$
\begin{aligned}
A_{r e g}^{\mu}= & A_{p}^{\mu} \\
+ & \frac{i g}{q^{2}+i q^{+} \epsilon} \int \frac{d^{2} p_{\perp}}{(2 \pi)^{2}}\left\{C_{U}^{\mu}\left(q, p_{\perp}\right)\left[\tilde{U}\left(k_{\perp}\right)-(2 \pi)^{2} \delta\left(k_{\perp}\right)\right]\right. \\
& \left.+C_{V, r e g}^{\mu}(q)\left[\tilde{V}\left(k_{\perp}\right)-(2 \pi)^{2} \delta\left(k_{\perp}\right)\right]\right\} \frac{\rho_{p}\left(p_{\perp}\right)}{p_{\perp}^{2}}
\end{aligned}
$$


where $\rho_{p}\left(p_{\perp}\right)$ is the color source distribution inside a proton, and $A_{p}^{\mu}$ is the gauge field created by the proton alone. In the MV model, it is given by,

$$
A_{p}^{\mu}=2 \pi g \delta^{\mu+} \delta\left(q^{-}\right) \frac{\rho_{p}\left(q_{\perp}\right)}{q_{\perp}^{2}},
$$

In second term of the formula 17, $p_{\perp}$ is the momentum carried by the incoming gluon from the proton and $k_{\perp}$ defined as $k_{\perp}=q_{\perp}-p_{\perp}$ is the momentum coming from the nucleus. For the polarized case, there exists a correlation between the transverse momentum $p_{\perp}$ and the transverse proton spin vector $S_{\perp}$. As shown below, such a correlation can be described by the ETQS function, and leads to a SSA for direct photon production. The four vectors $C_{U}^{\mu}\left(q, p_{\perp}\right)$ and $C_{V, r e g}^{\mu}$ are given by the following relations

$$
\begin{aligned}
& C_{U}^{+}\left(q, p_{\perp}\right)=-\frac{p_{\perp}^{2}}{q^{-}+i \epsilon}, \quad C_{U}^{-}\left(q, p_{\perp}\right)=\frac{k_{\perp}^{2}-q_{\perp}^{2}}{q^{+}+i \epsilon}, \quad C_{U}^{i}\left(q, p_{\perp}\right)=-2 \mathrm{p}_{\perp}^{i} \\
& C_{V, r e g}^{\mu}(q)=2 q^{\mu}-\delta^{-\mu} \frac{q^{2}}{q^{+}+i \epsilon}
\end{aligned}
$$

where the subscript ' $r e g^{\prime}$ indicates that the corresponding term of $A^{\mu}$ does not contain any $\delta\left(x^{+}\right)$ when expressed in coordinate space. Here, we specified the $q^{+}$pole structure according to the fact that this term arises from an initial state interaction. It is crucial to keep the imaginary part of this pole in order to generate the non-vanishing spin asymmetry. The notation $\mathrm{p}_{\perp}$ is used to denote four dimension vector with $p_{\perp}^{2}=-\mathrm{p}_{\perp}^{2} . \tilde{U}\left(k_{\perp}\right)$ and $\tilde{V}\left(k_{\perp}\right)$ are the Fourier transform of Wilson lines in the adjoint representation,

$$
\tilde{U}\left(k_{\perp}\right)=\int d^{2} x_{\perp} e^{i k_{\perp} \cdot x_{\perp}} \tilde{U}\left(x_{\perp}\right), \quad \tilde{V}\left(k_{\perp}\right)=\int d^{2} x_{\perp} e^{i k_{\perp} \cdot x_{\perp}} \tilde{V}\left(x_{\perp}\right)
$$

with

$$
\begin{aligned}
\tilde{U}\left(x_{\perp}\right) & =\mathcal{P} \exp \left[i g \int_{-\infty}^{+\infty} d z^{+} A_{A}^{-}\left(z^{+}, x_{\perp}\right) \cdot T\right], \\
\tilde{V}\left(x_{\perp}\right) & =\mathcal{P} \exp \left[i \frac{g}{2} \int_{-\infty}^{+\infty} d z^{+} A_{A}^{-}\left(z^{+}, x_{\perp}\right) \cdot T\right]
\end{aligned}
$$

where the $T$ are the generators of the adjoint representation. The singular terms reads,

$$
A_{\text {sing }}^{-}(q)=-\frac{i g}{q^{+}+i \epsilon} \int \frac{d^{2} p_{\perp}}{(2 \pi)^{2}}\left[\tilde{V}\left(k_{\perp}\right)-(2 \pi)^{2} \delta\left(k_{\perp}\right)\right] \frac{\rho_{p}\left(p_{\perp}\right)}{p_{\perp}^{2}}
$$

The peculiar Wilson line $\tilde{V}$ differs from the normal one $\tilde{U}$ by a factor $1 / 2$ in the exponent. It has been demonstrated that all terms containing $\tilde{V}$ cancel in the unpolarized amplitudes for gluon production and quark pair production in pA collisions [40,41. It will be shown below that the $\tilde{V}$ terms also drop out in the spin dependent amplitude for direct photon production.

Following the method outlined in Ref. [41, we calculate the contributions from the regular terms and the singular terms separately. Let us begin with the regular terms which do not contain a delta function $\delta\left(x^{+}\right)$. Their contributions are represented by the diagrams in Fig. 4 and Fig. 5. The amplitude from Fig. (4a) reads,

$$
\mathcal{M}_{4 a}=\left[i g A_{r e g}^{\mu}(q)\right]\left[\bar{u}\left(l_{q}\right) \gamma_{\mu} t^{a} S_{F}\left(x P-l_{q}\right)(i e) \notin u(x P)\right]
$$

where the momentum carried by the gluon produced through the fusion of a longitudinally polarized gluon from the proton and a small $\mathrm{x}$ gluons from the nucleus is given by $q=x_{g} P+p_{\perp}+x_{g}^{\prime} \bar{P}+k_{\perp}$. The 


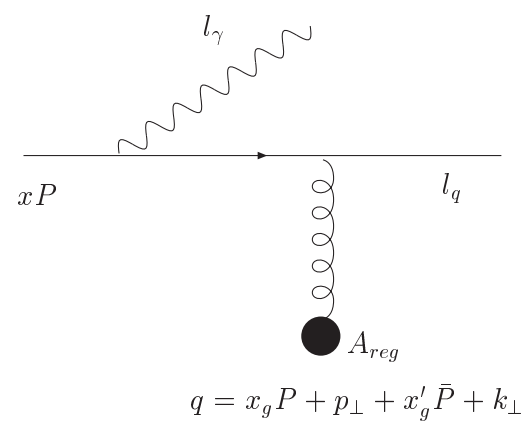

(a)

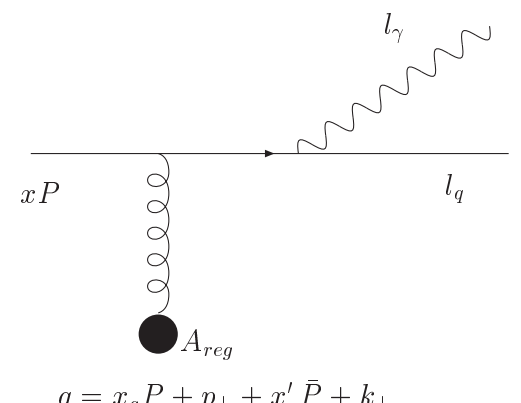

(b)

Figure 4: The contribution from the regular terms to the spin dependent amplitude. A black dot denotes a classical field $A_{\text {reg }}$ insertion.

soft gluon pole contribution to the amplitude $\mathcal{M}_{4 a}$ from the first term of Eq. (17) cancels between the diagram Fig. 4a and its mirror diagram in the same way as between the diagrams Fig. 11 and Fig. 11d. In addition, the contributions from the first part of $C_{V, \text { reg }}^{\mu}$ cancel between Fig. 4a and Fig. $4 \mathrm{~b}$ due to the Ward identity. The spin dependent amplitude thus can be explicitly written as,

$$
\begin{aligned}
& \mathcal{M}_{4 a}=-i e g^{2} \int \frac{d^{2} p_{\perp}}{(2 \pi)^{2}} \frac{\rho_{p, a}\left(p_{\perp}\right)}{p_{\perp}^{2}} \\
& \times \quad \bar{u}\left(l_{q}\right)\left\{\frac{\phi_{U}\left(q, p_{\perp}\right)}{q^{2}+i \epsilon} t^{b} S_{F}\left(x P-l_{q}\right) \notin\left[\tilde{U}\left(k_{\perp}\right)-(2 \pi)^{2} \delta\left(k_{\perp}\right)\right]_{b a}\right. \\
&\left.\quad-\frac{\not h}{x_{g} P+i \epsilon} t^{b} S_{F}\left(x P-l_{q}\right) \notin\left[\tilde{V}\left(k_{\perp}\right)-(2 \pi)^{2} \delta\left(k_{\perp}\right)\right]_{b a}\right\} u(x P)
\end{aligned}
$$

For the diagram in Fig. 4b, it is easy to verify that the first term of the Eq. (17) gives rise to the vanishing contribution. One thus obtains for the amplitude from Fig. $4 \mathrm{~b}$,

$$
\begin{aligned}
& \mathcal{M}_{4 b}=-i e g^{2} \int \frac{d^{2} p_{\perp}}{(2 \pi)^{2}} \frac{\rho_{p, a}\left(p_{\perp}\right)}{p_{\perp}^{2}} \\
& \times \quad \bar{u}\left(l_{q}\right)\left\{\notin S_{F}(x P+q) \frac{\phi_{U}\left(q, p_{\perp}\right)}{q^{2}+i \epsilon} t^{b}\left[\tilde{U}\left(k_{\perp}\right)-(2 \pi)^{2} \delta\left(k_{\perp}\right)\right]_{b a}\right. \\
&\left.\quad-\notin S_{F}(x P+q) \frac{\not h}{x_{g} P+i \epsilon} t^{b}\left[\tilde{V}\left(k_{\perp}\right)-(2 \pi)^{2} \delta\left(k_{\perp}\right)\right]_{b a}\right\} u(x P)
\end{aligned}
$$

The incoming quark also can directly interact with the classical gluon field from the nucleus. This is illustrated in Fig. 5. The first term of $A_{\text {reg }}$ does not contribute to the spin dependent part of the amplitudes from the diagrams in Fig. 5. One further notices that all of the $x_{g 1}^{\prime}$ poles in the amplitudes from the diagrams in Fig. 5d, Fig. 5e and Fig. 5f are lying in the same half plane. Therefore, after carrying out the $x_{g 1}^{\prime}$ integration using the theorem of the residues, one has

$$
\mathcal{M}_{5 d}=\mathcal{M}_{5 e}=\mathcal{M}_{5 f}=0
$$

We are left with the contributions from Fig. 5 a, Fig. 5 b and Fig. 55. After carrying out the $x_{g 1}^{\prime}$ integration, it becomes evident that the contributions from the first part of the $C_{V, r e g}^{\mu}$ term cancel 
between the diagrams in Fig. 5 a, Fig. 5b and Fig. 55. With these simplifications, the expression for the amplitude of Fig. 5 a is given by,

$$
\begin{aligned}
\mathcal{M}_{5 a}= & \int \frac{d^{4} k_{1}}{(2 \pi)^{4}} 2 \pi \delta\left(k_{1}^{+}\right)\left[i g A_{r e g}^{\mu}\left(q-k_{1}\right)\right] \\
& \times \bar{u}\left(l_{q}\right) \gamma_{\mu} t^{a} S_{F}\left(x P-l_{\gamma}+k_{1}\right) \not h\left[U\left(k_{1 \perp}\right)-(2 \pi)^{2} \delta\left(k_{1 \perp}\right)\right] S_{F}\left(x P-l_{\gamma}\right) i e \notin u(x P) \\
= & -i e g^{2} \int \frac{d k_{1}^{-} d^{2} k_{1 \perp}}{(2 \pi)^{3}} \int \frac{d^{2} p_{\perp}}{(2 \pi)^{2}} \frac{\rho_{p, a}\left(p_{\perp}\right)}{p_{\perp}^{2}} \bar{u}\left(l_{q}\right) \frac{\varphi_{U}\left(q-k_{1}, p_{\perp}\right)}{\left(q-k_{1}\right)^{2}+i \epsilon} t^{b} S_{F}\left(x P-l_{\gamma}+k_{1}\right) \not h \\
& \times\left[U\left(k_{1 \perp}\right)-(2 \pi)^{2} \delta\left(k_{1 \perp}\right)\right] S_{F}\left(x P-l_{\gamma}\right) \notin u(x P)\left[\tilde{U}\left(k_{\perp}-k_{1 \perp}\right)-(2 \pi)^{2} \delta\left(k_{\perp}-k_{1 \perp}\right)\right]_{b a} \\
+ & i e g^{2} \int \frac{d^{2} p_{\perp}}{(2 \pi)^{2}} \frac{\rho_{p, a}\left(p_{\perp}\right)}{p_{\perp}^{2}} \int d^{2} x_{\perp} e^{i k_{\perp} \cdot x_{\perp}} \bar{u}\left(l_{q}\right) \frac{\not h}{x_{g} P+i \epsilon} t^{b}\left[U\left(x_{\perp}\right)-1\right] S_{F}\left(x P-l_{\gamma}\right) \\
& \times \notin u(x P)\left[\tilde{V}\left(x_{\perp}\right)-1\right]_{b a}
\end{aligned}
$$

where we have applied the Eikonal approximation to the quark propagator $S_{F}\left(x P-l_{\gamma}+k_{1}\right)$ which appears in the hard part associated with the term containing $\left[\tilde{V}\left(x_{\perp}\right)-1\right]_{b a}$. The $k_{1}^{-}$and $k_{1 \perp}$ integrations have been carried out in the second term after making the Eikonal approximation. Following the similar procedure, we obtain the amplitude from the diagram in Fig. 5b,

$$
\begin{aligned}
\mathcal{M}_{5 b}= & -i e g^{2} \int \frac{d k_{1}^{-} d^{2} k_{1 \perp}}{(2 \pi)^{3}} \int \frac{d^{2} p_{\perp}}{(2 \pi)^{2}} \frac{\rho_{p, a}\left(p_{\perp}\right)}{p_{\perp}^{2}} \bar{u}\left(l_{q}\right) \notin S_{F}(x P+q) \frac{\phi_{U}\left(q-k_{1}, p_{\perp}\right)}{\left(q-k_{1}\right)^{2}+i \epsilon} t^{b} S_{F}\left(x P+k_{1}\right) \\
& \times \not h\left[U\left(k_{1 \perp}\right)-(2 \pi)^{2} \delta\left(k_{1 \perp}\right)\right] u(x P)\left[\tilde{U}\left(k_{\perp}-k_{1 \perp}\right)-(2 \pi)^{2} \delta\left(k_{\perp}-k_{1 \perp}\right)\right]_{b a} \\
+ & i e g^{2} \int \frac{d^{2} p_{\perp}}{(2 \pi)^{2}} \frac{\rho_{p, a}\left(p_{\perp}\right)}{p_{\perp}^{2}} \int d^{2} x_{\perp} e^{i k_{\perp} \cdot x_{\perp}} \bar{u}\left(l_{q}\right) \notin S_{F}(x P+q) \frac{\not h}{x_{g} P+i \epsilon} t^{b}\left[U\left(x_{\perp}\right)-1\right] u(x P) \\
& \times\left[\tilde{V}\left(x_{\perp}\right)-1\right]_{b a}
\end{aligned}
$$

The amplitude of the diagram in Fig. 5c does not receive any contribution from the second part of the $C_{V, \text { reg }}^{\mu}$ term since both $k_{1}^{-}$poles are lying in the same half plane. One thus obtains,

$$
\begin{aligned}
\mathcal{M}_{5 c}= & -i e g^{2} \int \frac{d k_{1}^{-} d^{2} k_{1 \perp}}{(2 \pi)^{3}} \int \frac{d^{2} p_{\perp}}{(2 \pi)^{2}} \frac{\rho_{p, a}\left(p_{\perp}\right)}{p_{\perp}^{2}} \bar{u}\left(l_{q}\right) \frac{\phi_{U}\left(q-k_{1}, p_{\perp}\right)}{\left(q-k_{1}\right)^{2}+i \epsilon} t^{b} S_{F}\left(x P-l_{\gamma}+k_{1}\right) \notin \\
& \times S_{F}\left(x P+k_{1}\right) \not \varkappa\left[U\left(k_{1 \perp}\right)-(2 \pi)^{2} \delta\left(k_{1 \perp}\right)\right] u(x P)\left[\tilde{U}\left(k_{\perp}-k_{1 \perp}\right)-(2 \pi)^{2} \delta\left(k_{\perp}-k_{1 \perp}\right)\right]_{b a} .
\end{aligned}
$$

We now turn to discuss the contributions from the singular terms. As explained in Ref. [41, it is convenient to compute it in coordinate space. The expression for the singular term in coordinate space is then given by [41],

$$
A_{\text {sing }}^{-}=-i \frac{g^{2}}{2}\left[A_{A}^{-}(x) \cdot T\right] \tilde{V}\left(x^{+},-\infty ; x_{\perp}\right) \theta\left(-x^{-}\right) \frac{1}{\nabla_{\perp}^{2}} \rho_{p}\left(x_{\perp}\right)
$$

where the theta function $\theta\left(-x^{-}\right)$reflects the fact that this gluon field is created through an initial state interaction. $\tilde{V}\left(x^{+},-\infty ; x_{\perp}\right)$ denotes an incomplete Wilson line:

$$
\tilde{V}\left(x^{+},-\infty ; x_{\perp}\right)=\mathcal{P} \exp \left[i \frac{g}{2} \int_{-\infty}^{x^{+}} d z^{+} A_{A}^{-}\left(z^{+}, x_{\perp}\right) \cdot T\right]
$$




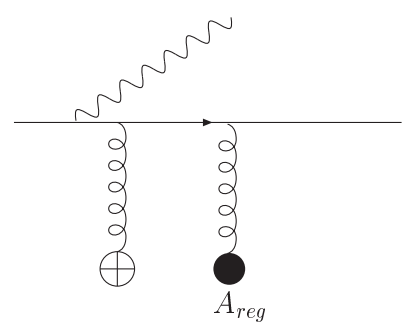

(a)

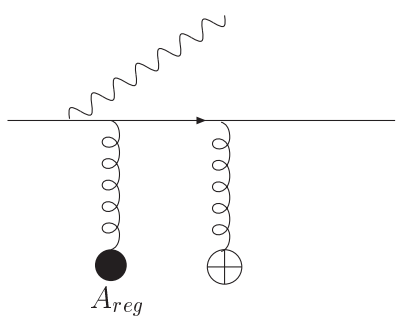

$(d)$

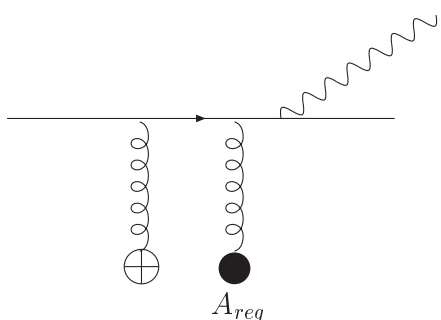

(b)

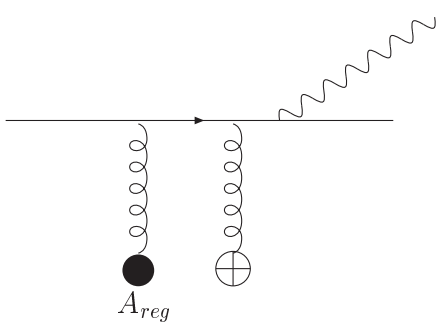

$(e)$

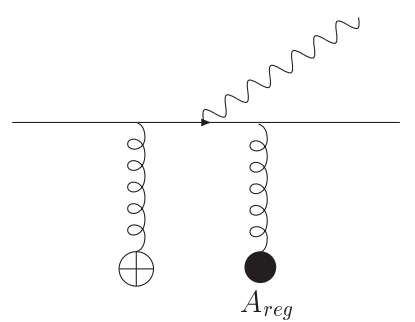

(c)

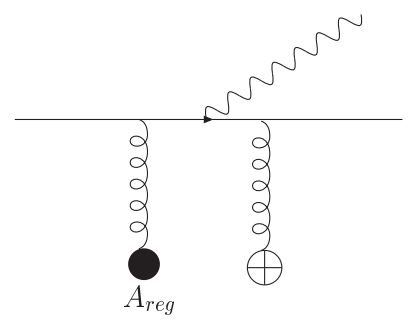

$(f)$

Figure 5: The contribution from the regular terms to the spin dependent amplitude. Black dot denotes a classical field $A_{\text {reg }}$ insertion, while the gluon line terminated by a cross surrounded with a circle denotes a classical field $A_{A}$ insertion.

In order to correctly compute the singular contributions, it is necessary to regularize $\delta\left(x^{+}\right)$by giving it a small width

$$
\delta\left(x^{+}\right) \longrightarrow \delta_{\epsilon}\left(x^{+}\right)
$$

where $\delta_{\epsilon}\left(x^{+}\right)$is a regular function whose support is $[0, \epsilon]$, which becomes $\delta\left(x^{+}\right)$when $\epsilon$ goes to zero. The final result is independent of the precise choice of the regularization. The field $A_{\text {sing }}^{\mu}$ is inserted on the quark line at the 'times' $x^{+}$, the incoming quark then rescatters off the field $A_{A}^{\mu}$ of the nucleus in the ranges $\left[0, x^{+}\right]$and $\left[x^{+}, \epsilon\right]$. The photon can only be emitted from the quark line either before multiple gluon re-scattering or after gluon re-scattering, because in the limit $\epsilon \rightarrow 0$, there is not sufficient time for emitting a photon inside the nucleus. The eight diagrams contributing to the amplitude are illustrated in Fig. 6. Combining the contributions from diagram Fig. 6a, Fig. 6b, Fig. 6rc and Fig. 6f, the resulting amplitude in coordinate space is,

$$
\begin{aligned}
\mathcal{M}_{6 a+6 b+6 c+6 d}= & \int d^{4} x e^{i q \cdot x} \bar{u}\left(l_{q}\right) U\left(+\infty, x^{+} ; x_{\perp}\right) \not h\left[i g t^{a} A_{\text {sing }}^{-a}(x)\right] S_{F}\left(x P-l_{\gamma}\right) \\
& \times i e \notin U\left(x^{+},-\infty ; x_{\perp}\right) u(x P) \\
= & -e g \int d^{4} x e^{i q \cdot x} \bar{u}\left(l_{q}\right) U\left(+\infty, x^{+} ; x_{\perp}\right) \not h\left[t^{a} A_{\text {sing }}^{-a}(x)\right] S_{F}\left(x P-l_{\gamma}\right) \\
& \times \notin U^{\dagger}\left(+\infty, x^{+} ; x_{\perp}\right) U\left(+\infty,-\infty ; x_{\perp}\right) u(x P)
\end{aligned}
$$

where the incomplete Wilson lines in the fundamental representation are defined as

$$
\begin{aligned}
& U\left(+\infty, x^{+} ; x_{\perp}\right)=\mathcal{P} \exp \left[i g \int_{x^{+}}^{+\infty} d z^{+} A_{A}^{-}\left(z^{+}, x_{\perp}\right) \cdot t\right] \\
& U\left(x^{+},-\infty ; x_{\perp}\right)=\mathcal{P} \exp \left[i g \int_{-\infty}^{x^{+}} d z^{+} A_{A}^{-}\left(z^{+}, x_{\perp}\right) \cdot t\right]
\end{aligned}
$$




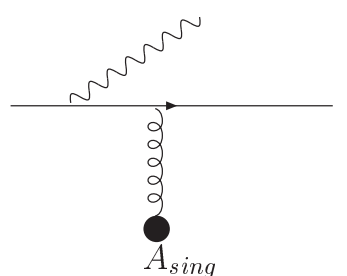

(a)

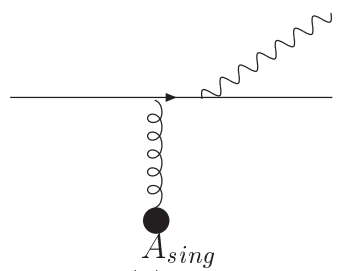

(e)

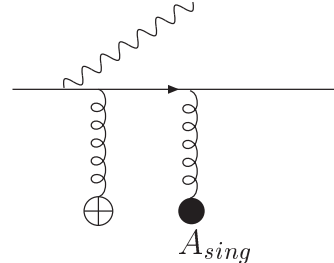

(b)

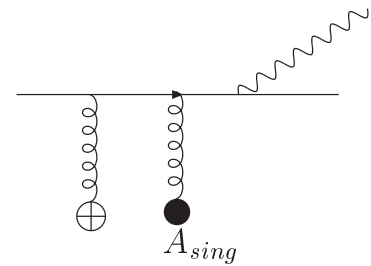

$(f)$

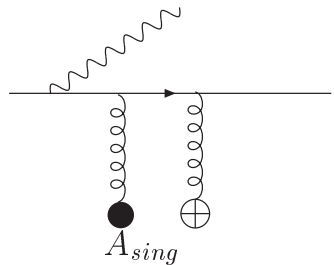

$(c)$

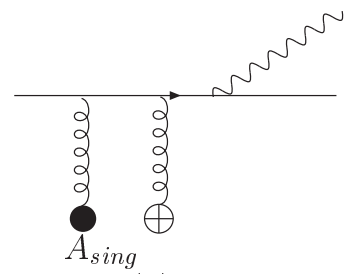

$(g)$

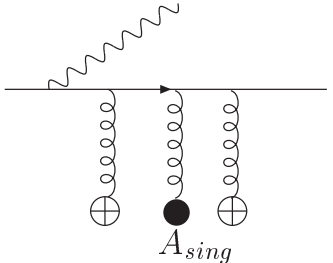

$(d)$

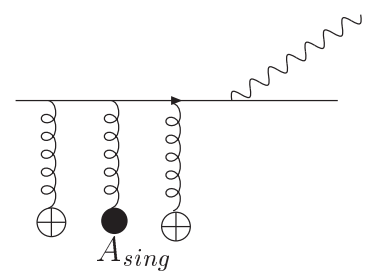

$(h)$

Figure 6: The contribution from the singular terms to the spin dependent amplitude. A black dot denotes a classical field $A_{\text {sing }}$ insertion, while the gluon line terminated by a cross surrounded with a circle denotes a classical field $A_{A}$ insertion.

In order to simplify this expression, we use the algebraic identity,

$$
U\left(+\infty, x^{+} ; x_{\perp}\right) t^{a} U^{\dagger}\left(+\infty, x^{+} ; x_{\perp}\right)=t^{b} \tilde{U}_{b a}\left(+\infty, x^{+} ; x_{\perp}\right)
$$

and also the formula first derived in Ref. [41],

$$
i \frac{g}{2} \int_{-\infty}^{+\infty} d x^{+} \tilde{U}\left(+\infty, x^{+} ; x_{\perp}\right)\left[A_{A}^{-}(x) \cdot T\right] \tilde{V}\left(x^{+},-\infty ; x_{\perp}\right)=\tilde{U}\left(x_{\perp}\right)-\tilde{V}\left(x_{\perp}\right)
$$

After carrying out the $x^{-}$and $x^{+}$integrations, the above expression is simplified to,

$$
\begin{aligned}
\mathcal{M}_{6 a+6 b+6 c+6 d}= & i e g^{2} \int \frac{d^{2} p_{\perp}}{(2 \pi)^{2}} \frac{\rho_{p, a}\left(p_{\perp}\right)}{p_{\perp}^{2}} \int d^{2} x_{\perp} e^{i k_{\perp} \cdot x_{\perp}} \\
& \times \bar{u}\left(l_{q}\right) \not h t^{b} S_{F}\left(x P-l_{\gamma}\right) \notin U\left(x_{\perp}\right) u(x P) \frac{1}{x_{g} P+i \epsilon}\left[\tilde{U}\left(x_{\perp}\right)-\tilde{V}\left(x_{\perp}\right)\right]_{b a}
\end{aligned}
$$

Following the same procedure, it is straightforward to write down the amplitude from the diagrams in Fig. 6r, Fig. 6f, Fig. 6r and Fig. 6h

$$
\begin{aligned}
\mathcal{M}_{6 e+6 f+6 g+6 h}= & i e g^{2} \int \frac{d^{2} p_{\perp}}{(2 \pi)^{2}} \frac{\rho_{p, a}\left(p_{\perp}\right)}{p_{\perp}^{2}} \int d^{2} x_{\perp} e^{i k_{\perp} \cdot x_{\perp}} \\
& \times \bar{u}\left(l_{q}\right) \notin S_{F}(x P+q) \not h t^{b} U\left(x_{\perp}\right) u(x P) \frac{1}{x_{g} P+i \epsilon}\left[\tilde{U}\left(x_{\perp}\right)-\tilde{V}\left(x_{\perp}\right)\right]_{b a}
\end{aligned}
$$

Collecting all pieces together, the total amplitude reads,

$$
\begin{aligned}
\mathcal{M}_{4+5+6}= & -i e g^{2} \int \frac{d^{2} p_{\perp}}{(2 \pi)^{2}} \frac{\rho_{p, a}\left(p_{\perp}\right)}{p_{\perp}^{2}} \int \frac{d k_{1}^{-} d^{2} k_{1 \perp}}{(2 \pi)^{3}} \bar{u}\left(l_{q}\right) \\
& \times\left\{\notin S_{F}(x P+q) \frac{\phi_{U}\left(q-k_{1}, p_{\perp}\right)}{\left(q-k_{1}\right)^{2}+i \epsilon} t^{b} S_{F}\left(x P+k_{1}\right) \not h U\left(k_{1 \perp}\right)\right.
\end{aligned}
$$




$$
\begin{aligned}
&+ \frac{\phi_{U}\left(q-k_{1}, p_{\perp}\right)}{\left(q-k_{1}\right)^{2}+i \epsilon} t^{b} S_{F}\left(x P-l_{\gamma}+k_{1}\right) \not u\left(k_{1 \perp}\right) S_{F}\left(x P-l_{\gamma}\right) \notin \\
&+\left.\frac{\phi_{U}\left(q-k_{1}, p_{\perp}\right)}{\left(q-k_{1}\right)^{2}+i \epsilon} t^{b} S_{F}\left(x P-l_{\gamma}+k_{1}\right) \notin S_{F}\left(x P+k_{1}\right) \not U\left(k_{1 \perp}\right)\right\} \\
& \times u(x P)\left[\tilde{U}\left(k_{\perp}-k_{1 \perp}\right)-(2 \pi)^{2} \delta\left(k_{\perp}-k_{1 \perp}\right)\right]_{b a} \\
&+i e g^{2} \int \frac{d^{2} p_{\perp}}{(2 \pi)^{2}} \frac{\rho_{p, a}\left(p_{\perp}\right)}{p_{\perp}^{2}} \int d^{2} x_{\perp} e^{i k_{\perp} \cdot x_{\perp}} \\
& \times \bar{u}\left(l_{q}\right) \frac{\not h S_{F}\left(x P-l_{\gamma}\right) \notin+\notin S_{F}(x P+q) \not h}{x_{g} P+i \epsilon} t^{b} U\left(x_{\perp}\right) u(x P)\left[\tilde{U}\left(x_{\perp}\right)-1\right]_{b a}
\end{aligned}
$$

where the $\tilde{V}\left(x_{\perp}\right)$ terms drop out as expected. The spin dependence of the total amplitude comes from the correlation between $p_{\perp}$ and the transverse spin vector of the proton $S_{\perp}$. With the obtained amplitude, we are ready to compute the twist-3 spin dependent cross section. However, since the calculation of the full polarized cross section is quite involved, we restrict ourself here to the discussion of two results, namely the derivative term contribution in a dense medium and the cross section in the large photon transverse momentum limit. We believe that it is sufficient to demonstrate the most interesting feature of the complete result, on the one hand, and to check the consistence of our formalism by extrapolating the result to the high transverse momentum limit and comparing it with the polarized cross section computed in the collinear twist-3 approach, on the other hand.

\subsection{The derivative term in a dense medium}

One of the terms in the polarized cross section proportional to the derivative of the ETQS function is often refereed to as the derivative term, which is usually considered to be the dominant contribution to the spin asymmetry in the forward region. In this subsection, we sketch a few key steps when deriving the expression for the derivative term. As mentioned in the previous section, the spin dependent hard part is calculated from an interference of two partonic scattering amplitudes, as illustrated in Fig. 3. We thus proceed by defining a hard part $H_{B o r n}^{\mu \nu}\left(p_{\perp}, k_{\perp}\right)$ according to the following equation,

$$
\begin{gathered}
\left.\sum_{\text {spins,color }} \frac{1}{2 x} \mathcal{M}_{4+5+6}\right|_{\text {derivative }} \mathcal{M}_{3}^{*} \delta\left(l_{q}^{2}\right)=\int \frac{d^{2} p_{\perp}}{(2 \pi)^{2}} \frac{1}{x_{g} P+i \epsilon} H_{B o r n}^{\mu \nu}\left(p_{\perp}, k_{\perp}\right) n_{\mu} n_{\nu} \delta\left(l_{q}^{2}\right) \\
\times \int d^{2} x_{\perp} d^{2} y_{\perp} e^{i k_{\perp} \cdot\left(x_{\perp}-y_{\perp}\right)} \operatorname{Tr}_{\mathrm{c}}\left\{\left[U^{\dagger}\left(y_{\perp}\right)-1\right] t^{b} U\left(x_{\perp}\right) \frac{\rho_{p, a}\left(p_{\perp}\right)}{p_{\perp}^{2}}\right\}\left[\tilde{U}\left(x_{\perp}\right)-1\right]_{b a}
\end{gathered}
$$

where $\left.\mathcal{M}_{4+5+6}\right|_{\text {derivative }}$ represents the last term in Eq. (42). As we shall explain below, the $C_{U}$ terms in Eq. (42) do not give rise to a derivative term contribution. The next step is to expand the hard part in terms of $p_{\perp}$,

$$
H_{\text {Born }}^{\mu \nu}\left(p_{\perp}, k_{\perp}\right) \delta\left(l_{q}^{2}\right)=\left.H_{\text {Born }}^{\mu \nu}\left(p_{\perp}, k_{\perp}\right) \delta\left(l_{q}^{2}\right)\right|_{p_{\perp}=0}+\left.\frac{\partial H_{B o r n}^{\mu \nu}\left(p_{\perp}, k_{\perp}\right) \delta\left(l_{q}^{2}\right)}{\partial p_{\perp}^{\rho}}\right|_{p_{\perp}=0} p_{\perp}^{\rho}+\ldots
$$

where the spin dependent part is the term linear in $p_{\perp}$, in which we only keep the contribution containing the derivative of the delta function, leading to a derivative of the ETQS function by partial integration. More precisely, the relevant contribution is given by,

$$
\left[\frac{\left(k_{\perp}^{\rho}-l_{\gamma \perp}^{\rho}\right)}{l_{q} \cdot P} H_{B o r n}^{\mu \nu}\left(p_{\perp}, k_{\perp}\right) \frac{\partial \delta\left(l_{q}^{2}\right)}{\partial x}\right]_{p_{\perp}=0} p_{\perp, \rho}
$$


Once the collinear expansion has been carried out, $p_{\perp}$ is set to zero in the hard part as indicated in the above formula. At this point, it becomes clear why the $C_{U}$ terms do not give rise to a derivative term contribution, namely simply because $C_{U}$ vanishes when $p_{\perp}=0$,

$$
C_{U}^{\mu}\left(q-k_{1}, p_{\perp}=0\right)=0
$$

We proceed by combining $p_{\perp}$ with the color source term to yield the gluon field strength operator using Eq. (18),

$$
\frac{\rho_{p, a}\left(p_{\perp}\right)}{p_{\perp}^{2}} p_{\perp, \rho} \longrightarrow F_{\rho, a}^{+}\left(p_{\perp}\right)
$$

Since the hard part is independent of $p_{\perp}$ after the collinear expansion, the $p_{\perp}$ integration can be trivially carried out. The quark gluon correlator can subsequently be parameterized through the ETQS function,

$$
\int \frac{d^{2} p_{\perp}}{(2 \pi)^{2}}\left\langle\bar{\psi} \frac{\rho_{p, a}\left(p_{\perp}\right)}{p_{\perp}^{2}} p_{\perp, \rho} \psi\right\rangle_{\text {proton }} \longrightarrow \epsilon^{\rho S_{\perp} n p} \frac{2}{N_{c}^{2}-1} \frac{1}{2 \pi} t^{a} T_{F}\left(x, x+x_{g}\right)
$$

where the correlation between the transverse spin vector of the proton and $p_{\perp}$ becomes manifest. The color structure associated with the ETQS function is fixed by following the argument made in Refs. 6, 7, 10. Moreover, one needs to isolate the imaginary part of the soft gluon pole using the identity $\frac{1}{x_{g} P+i \epsilon}=\mathrm{P} \frac{1}{x_{g} P}-i \pi \delta\left(x_{g} P\right)$. The contributions from its real part cancel out between mirror diagrams. With all these calculation recipes, one can readily compute the contribution from the derivative term. The spin dependent cross section involving the derivative term takes the following form,

$$
\begin{aligned}
\frac{d^{3} \Delta \sigma}{d^{2} l_{\gamma \perp} d z} \propto & \int d^{2} k_{\perp} d x_{g}^{\prime} d x\left[\epsilon^{l_{\gamma} S_{\perp} n p}-\epsilon^{\mathrm{k}_{\perp} S_{\perp} n p}\right] \frac{1}{l_{q} \cdot P}\left[H_{B o r n}^{\mu \nu}\left(p_{\perp}, k_{\perp}\right) \frac{\partial \delta\left(l_{q}^{2}\right)}{\partial x}\right]_{p_{\perp}=0} \sum_{q} e_{q}^{2} T_{F, q}(x, x) \\
& \times \int d^{2} x_{\perp} d^{2} y_{\perp} e^{i k_{\perp} \cdot\left(x_{\perp}-y_{\perp}\right)}\left\langle\operatorname{Tr}_{\mathrm{c}}\left[\left(U^{\dagger}\left(y_{\perp}\right)-1\right) t^{b} U\left(x_{\perp}\right) t^{a}\right]\left[\tilde{U}\left(x_{\perp}\right)-1\right]_{b a}\right\rangle_{x_{g}^{\prime}}
\end{aligned}
$$

The expression for the soft part from the nucleus side in the above formula can be further simplified. Using Eq. (38), one obtains,

$$
\begin{aligned}
& \operatorname{Tr}_{\mathrm{c}}\left[\left(U^{\dagger}\left(y_{\perp}\right)-1\right) t^{b} U\left(x_{\perp}\right) t^{a}\right]\left[\tilde{U}\left(x_{\perp}\right)-1\right]_{b a} \\
& =C_{F} \operatorname{Tr}_{\mathrm{c}}\left[U^{\dagger}\left(y_{\perp}\right) U\left(x_{\perp}\right)\right]-\operatorname{Tr}_{\mathrm{c}}\left[U^{\dagger}\left(y_{\perp}\right) t^{a} U\left(x_{\perp}\right) t^{a}\right]
\end{aligned}
$$

Employing the Fierz identity,

$$
t_{i j}^{a} t_{k l}^{a}=\frac{1}{2} \delta_{i l} \delta_{k j}-\frac{1}{2 N_{c}} \delta_{i j} \delta_{k l}
$$

the last term in Eq. (50) is rewritten as,

$$
\operatorname{Tr}_{\mathrm{c}}\left[U^{\dagger}\left(y_{\perp}\right) t^{a} U\left(x_{\perp}\right) t^{a}\right]=\frac{1}{2} \operatorname{Tr}_{\mathrm{c}}\left[U^{\dagger}\left(y_{\perp}\right)\right] \operatorname{Tr}_{\mathrm{c}}\left[U\left(x_{\perp}\right)\right]-\frac{1}{2 N_{c}} \operatorname{Tr}_{\mathrm{c}}\left[U^{\dagger}\left(y_{\perp}\right) U\left(x_{\perp}\right)\right]
$$

Inserting the above decomposition into Eq. (50), we obtain,

$$
\begin{aligned}
& \operatorname{Tr}_{\mathrm{c}}\left[U^{\dagger}\left(y_{\perp}\right) t^{b} U\left(x_{\perp}\right) t^{a}\right]\left[\tilde{U}\left(x_{\perp}\right)-1\right]_{b a} \\
= & \frac{N_{c}}{2} \operatorname{Tr}_{\mathrm{c}}\left[U^{\dagger}\left(y_{\perp}\right) U\left(x_{\perp}\right)\right]-\frac{1}{2} \operatorname{Tr}_{\mathrm{c}}\left[U^{\dagger}\left(y_{\perp}\right)\right] \operatorname{Tr}_{\mathrm{c}}\left[U\left(x_{\perp}\right)\right]
\end{aligned}
$$


where the first term can be related to the dipole type gluon distribution, while the second term is a new contribution that arises from the color entanglement effect. To arrive at the final expression for the polarized cross section, we need to explicitly evaluate the hard part and carry out the $x_{g}^{\prime}$ integration using the delta function $\delta\left(l_{q}^{2}\right)$ which originates from the on shell condition. After combining the contributions from the left and right cut diagrams, one ends up with,

$$
\begin{aligned}
\frac{d^{3} \Delta \sigma}{d^{2} l_{\gamma \perp} d z}= & \frac{\alpha_{s} \alpha_{e m} N_{c}}{N_{c}^{2}-1} \frac{1+(1-z)^{2}}{z l_{\gamma \perp}^{2}}(z-1) \int_{x_{m i n}}^{1} d x \int d^{2} k_{\perp} \frac{\left[\epsilon^{l_{\gamma} S_{\perp} n p}-\epsilon^{\mathrm{k}_{\perp} S_{\perp} n p}\right]}{\left(k_{\perp}-l_{\gamma \perp} / z\right)^{2}\left(k_{\perp}-l_{\gamma \perp}\right)^{2}} \\
& \times \sum_{q} e_{q}^{2}\left[-x \frac{d}{d x} T_{F, q}(x, x)\right]\left[x_{g}^{\prime} G_{D P}\left(x_{g}^{\prime}, k_{\perp}\right)-x_{g}^{\prime} G_{4}\left(x_{g}^{\prime}, k_{\perp}\right)\right]
\end{aligned}
$$

which is the main result of this section. Here we introduce a new gluon distribution $G_{4}\left(x_{g}^{\prime}, k_{\perp}\right)$. It is defined as,

$$
x_{g}^{\prime} G_{4}\left(x_{g}^{\prime}, k_{\perp}\right)=\frac{k_{\perp}^{2} N_{c}}{2 \pi^{2} \alpha_{s}} \int \frac{d^{2} x_{\perp} d^{2} y_{\perp}}{(2 \pi)^{2}} e^{i k_{\perp} \cdot\left(x_{\perp}-y_{\perp}\right)} \frac{1}{N_{c}^{2}}\left\langle\operatorname{Tr}_{\mathrm{c}}\left[U\left(x_{\perp}\right)\right] \operatorname{Tr}_{\mathrm{c}}\left[U^{\dagger}\left(y_{\perp}\right)\right]\right\rangle_{x_{g}^{\prime}}
$$

This new gluon distribution only shows up in the spin dependent cross section and is absent in the unpolarized cross section. The extra gluon exchange between the remnant of the proton and active partons plays a crucial role in yielding the nontrivial Wilson line structure in the Eq. (566). The additional term associated with $G_{4}\left(x_{g}^{\prime}, k_{\perp}\right)$ thus essentially arises from the color entanglement effect.

More interestingly, the gluon distribution $G_{4}\left(x_{g}^{\prime}, k_{\perp}\right)$ can be calculated in the MV model through a recursion procedure systemically developed in Ref. [41]. In the MV model, to our surprise, it is simply given by,

$$
\begin{aligned}
x_{g}^{\prime} G_{4}\left(x_{g}^{\prime}, k_{\perp}\right) & =\frac{k_{\perp}^{2} N_{c}}{2 \pi^{2} \alpha_{s}} \pi R_{0}^{2} \int \frac{d^{2} r_{\perp}}{(2 \pi)^{2}} e^{i k_{\perp} \cdot r_{\perp}} \frac{1}{N_{c}^{2}} e^{-\frac{1}{4} r_{\perp}^{2} Q_{s q}^{2}} \\
& =\frac{1}{N_{c}^{2}} G_{D P}\left(x_{g}^{\prime}, k_{\perp}\right)
\end{aligned}
$$

We thus conclude that the novel gluon distribution $G_{4}$ is sizable, though it is suppressed in the large $N_{c}$ limit as compared to the dipole type gluon distribution.

We now make some observations on the polarized cross section in the different kinematic limits. At small photon transverse momentum $\Lambda_{Q C D} \ll l_{\gamma \perp} \ll Q_{s q} \sim k_{\perp}$, the denominator in the Eq. (54) can be approximated as,

$$
\frac{1}{\left(k_{\perp}-l_{\gamma \perp} / z\right)^{2}\left(k_{\perp}-l_{\gamma \perp}\right)^{2}} \approx\left(1+\frac{1+z}{z} \frac{2 k_{\perp} \cdot l_{\gamma \perp}}{k_{\perp}^{2}}\right) \frac{1}{k_{\perp}^{4}}
$$

Once adopting such approximation in the both unpolarized cross section and polarized cross section, it is easy to see that the spin asymmetry computed in the hybrid approach scales as $l_{\perp}$ at low transverse momentum. As a comparison, in the standard collinear twist-3 framework, the predicated spin asymmetry is proportional to $1 / l_{\perp}$. For $l_{\gamma \perp} \gg Q_{s q} \sim k_{\perp}$, the denominator can be approximated by $1 /\left(k_{\perp}-l_{\gamma \perp} / z\right)^{2} \approx z^{2} / l_{\gamma \perp}^{2}$. Using Eq. 15 and Eq. 56, the polarized cross section is correspondingly reduced to,

$$
\begin{aligned}
\frac{d^{3} \Delta \sigma}{d^{2} l_{\gamma \perp} d z}= & \frac{\alpha_{s} \alpha_{e m} N_{c}}{N_{c}^{2}-1} \epsilon^{l_{\gamma} S_{\perp} n p} \frac{z\left[1+(1-z)^{2}\right]}{l_{\gamma \perp}^{6}}(z-1) \\
& \times \sum_{q} e_{q}^{2} \int_{x_{\min }}^{1} d x\left[-x \frac{d}{d x} T_{F, q}(x, x)\right]\left\{x_{g}^{\prime} G\left(x_{g}^{\prime}\right)-\frac{1}{N_{c}^{2}} x_{g}^{\prime} G\left(x_{g}^{\prime}\right)\right\}
\end{aligned}
$$


which recovers the result for the derivative term contribution computed in the collinear approach if one ignores the second term of the soft parts that arises from color entanglement effect. In the next subsection, we show that the non-derivative term contribution also can be reproduced in our hybrid formalism in the kinematical limit where $l_{\gamma \perp} \gg Q_{s q} \sim k_{\perp}$ provided that the $G_{4}$ contribution is neglected.

We now close this subsection with a few further remarks.

- The color entanglement effect discovered for double spin asymmetries (DSA) leads to a violation of generalized TMD factorization [26]. In contrast, the process we study is factorizable though there exists an additional term arises from the color entanglement effect. The reason is that collinear factorization is applied on the proton side and the basic building block of the soft part on the nucleus side, namely the Wilson line, is a universal object.

- Apparently, the gluon distribution $G_{4}\left(x_{g}^{\prime}, k_{\perp}\right)$ vanishes in the single gluon exchange approximation, thus requireing at least two gluon exchange. This is in line with the argument that two extra gluon attachments from an unpolarized target are required to generate a non-trivial color entanglement effect [26].

- Since $G_{4}\left(x_{g}^{\prime}, k_{\perp}\right)$ can be explicitly evaluated in the MV model, one can test it by measuring SSAs for photon production in $\mathrm{p}^{\uparrow} \mathrm{A}$ collisions.

- In general color entanglement plays a less important role for $\mathrm{p}^{\uparrow} \mathrm{p}$ collisions than for $\mathrm{p}^{\uparrow} \mathrm{A}$ collisions as the existence of $G_{4}\left(x_{g}^{\prime}, k_{\perp}\right)$ requires at least two gluons exchange. However, the SSA for photon production in $\mathrm{p}^{\uparrow} \mathrm{p}$ collisions might receive the significant contribution from the additional term proportional to the distribution $G_{4}\left(x_{g}^{\prime}, k_{\perp}\right)$ in the very forward region.

- If we apply collinear factorization on both the proton and nucleus sides, according to the model calculation result Eq. 56, the color entanglement effect would survive. This might indicate that the collinear higher-twist factorization breaks down in the process we study.

\subsection{The collinear limit}

In this subsection, we show that not only the derivative term contribution but also the non-derivative term contribution computed in the collinear factorization framework can be recovered from our hybrid approach in the limit $l_{\gamma \perp} \gg Q_{s q}$ after neglecting the additional contribution results from color entanglement effect. To achieve this goal, our main strategy is to systematically neglect all terms suppressed by powers of $Q_{s q} / l_{\gamma \perp}\left(\right.$ or $\left.k_{\perp} / l_{\gamma \perp}\right)$.

The first step is to set $k_{1 \perp}=0$ in the hard part in Eq. (42). This is a well justified approximation in the kinematical limit that we consider because the typical transverse momentum carried by small x gluons is of the order of $Q_{s q}$ and thus much smaller than the photon transverse momentum $l_{\gamma \perp}$. We then can trivially carry out the $k_{1 \perp}$ and $k_{1}^{-}$integrations in Eq. (42) after applying the Eikonal approximation to the quark propagators $S_{F}\left(x P+k_{1}\right)$ and $S_{F}\left(x P-l_{\gamma}+k_{1}\right)$. As a result, the polarized amplitude simplifies to,

$$
\begin{aligned}
& \mathcal{M}_{4+5+6} \approx-i e g^{2} \int \frac{d^{2} p_{\perp}}{(2 \pi)^{2}} \frac{\rho_{p, a}\left(p_{\perp}\right)}{p_{\perp}^{2}} \int d^{2} x_{\perp} e^{i k_{\perp} \cdot x_{\perp}} \bar{u}\left(l_{q}\right) \\
& \times \frac{\phi_{L}\left(q, p_{\perp}\right) S_{F}\left(x P-l_{\gamma}\right) \notin+\notin S_{F}(x P+q) \phi_{L}\left(q, p_{\perp}\right)}{q^{2}+i \epsilon} t^{b} U\left(x_{\perp}\right) u(x P)\left[\tilde{U}\left(x_{\perp}\right)-1\right]_{b a}
\end{aligned}
$$


where $\not_{L}$ is the well known effective Lipatov vertex for the production of a gluon via the fusion of two gluons. It is given by,

$$
\begin{aligned}
\frac{\phi_{L}\left(q, p_{\perp}\right)}{q^{2}+i \epsilon} & =\frac{\phi_{U}\left(q, p_{\perp}\right)}{q^{2}+i \epsilon}-\frac{\not h}{q^{+}+i \epsilon} \\
& =\frac{-p_{\perp}^{2}}{\left(q^{2}+i \epsilon\right)\left(q^{-}+i \epsilon\right)} \not p+\frac{k_{\perp}^{2}}{\left(q^{2}+i \epsilon\right)\left(q^{+}+i \epsilon\right)} \not h-2 \frac{q^{-} \not h+p_{\perp}}{q^{2}+i \epsilon}
\end{aligned}
$$

One notices that the first term in the above formula can be neglected since it is beyond the order in $p_{\perp}$ that we consider. The second term contains one soft gluon pole and one hard gluon pole,

$$
\frac{1}{q^{+}+i \epsilon} \longrightarrow x_{g}=0, \quad \frac{1}{q^{2}+i \epsilon} \longrightarrow x_{g}=\frac{\left(k_{\perp}+p_{\perp}\right)^{2}}{2 x_{g}^{\prime} P \cdot \bar{P}}
$$

When the photon transverse momentum is much larger than the saturation scale, the hard gluon pole $x_{g}=\left(k_{\perp}+p_{\perp}\right)^{2} /\left(2 x_{g}^{\prime} P \cdot \bar{P}\right) \approx 0$ degenerates with the soft gluon pole. It is easy to verify that the contributions from both cancel in this kinematical limit. We are thus left with the last term. Using the Ward identity, it can be replaced with

$$
-2 \frac{q^{-} \not h+\mathrm{p}_{\perp}}{q^{2}+i \epsilon} \longrightarrow 2 \frac{q^{+} \not p+\mathrm{l}_{\perp}}{q^{2}+i \epsilon}
$$

Applying the Ward identity to the right side of the cut diagrams, we may make the following replacement for the gluon polarization vector,

$$
n^{\mu} \longrightarrow \frac{-\mathrm{k}_{\perp}^{\mu}}{q^{-}}
$$

With these replacements, the hard part can be written as,

$$
-\frac{1}{q^{-}} \delta\left(q^{2}\right)\left(x_{g} P_{\mu}+\mathrm{k}_{\perp, \mu}\right) \mathrm{k}_{\perp, \nu} H_{B o r n}^{\mu \nu}\left(p_{\perp}, k_{\perp}\right) \delta\left(l_{q}^{2}\right)
$$

where $H_{\text {Born }}^{\mu \nu}\left(p_{\perp}, k_{\perp}\right)$ has been defined in the previous subsection. To proceed further, we keep the leading term which is proportional to $k_{\perp}^{2}$ and neglect all higher order terms in $k_{\perp}$. After averaging over the azimuthal angle of $k_{\perp}$, the hard part reads,

$$
\frac{1}{2 q^{-}} k_{\perp}^{2}\left\{\frac{P_{\mu} \mathrm{p}_{\perp, \nu}}{x_{g}^{\prime} P \cdot \bar{P}}+d_{\perp, \mu \nu}\right\}\left[H_{B o r n}^{\mu \nu}\left(p_{\perp}, k_{\perp}\right) \delta\left(l_{q}^{2}\right) \delta\left(q^{2}\right)\right]_{k_{\perp}=0}
$$

where the tensor $d_{\perp, \mu \nu}$ is defined as $d_{\perp, \mu \nu}=-g_{\mu \nu}+\left(p_{\mu} n_{\nu}+p_{\nu} n_{\mu}\right) / p \cdot n$. $k_{\perp}^{2}$ in the above formula can be combined with the soft part, namely the Wilson lines, and related to the transverse momentum dependent gluon distributions $G_{D P}\left(x_{g}^{\prime}, k_{\perp}\right)$ and $G_{4}\left(x_{g}^{\prime}, k_{\perp}\right)$. Since there is no $k_{\perp}$ dependence in the hard part any longer, the $k_{\perp}$ integration can be trivially carried out using the relations presented in Eq. (15) and Eq. (56). The resulting soft part is simply the ordinary integrated gluon distribution if we ignore the term generated from $G_{4}$.

On the other hand, the spin dependent hard part from Fig. 19 calculated in the collinear approach is proportional to the following expression

$$
\frac{1}{2} d_{\perp, \nu}^{\sigma}\left[H_{\text {Born }}^{\mu \nu}\left(p_{\perp}, k_{\perp}\right) \delta\left(l_{q}^{2}\right) \delta\left(q^{2}\right)\right]_{k_{\perp}=0} \Lambda_{\sigma \rho \mu} p^{\rho}
$$


with the three gluon vertex being defined as,

$$
\begin{aligned}
\Lambda_{\sigma \rho \mu} & =g_{\sigma \rho}\left(x_{g}^{\prime} \bar{P}-x_{g} P-\mathrm{p}_{\perp}\right)_{\mu}+g_{\rho \mu}\left(2 x_{g} P+2 \mathrm{p}_{\perp}+x_{g}^{\prime} \bar{P}\right)_{\sigma} \\
& +g_{\mu \sigma}\left(-2 x_{g}^{\prime} \bar{P}-x_{g} P-\mathrm{p}_{\perp}\right)_{\rho}
\end{aligned}
$$

After few algebra steps, one finds that the expressions (65) and (66) for the hard parts derived in the two different formalisms agree with each other up to some trivial pre-factor. Following the procedure outlined above, one can show that both formalisms also yield the same hard parts for the mirror diagrams. Therefore, we confirmed that our result without the $G_{4}$ related contribution being included reduces to that computed in the standard collinear approach in the kinematical region $l_{\gamma \perp} \gg Q_{s q}$. We consider this as an important consistency check for the hybrid approach.

\section{Summary}

We studied the SSA in direct photon production in polarized $\mathrm{p}^{\uparrow} \mathrm{A}$ collisions. The calculation is carried out using a hybrid approach in which the nucleus is treated in the CGC framework while the collinear twist-3 formalism is applied on the proton side. We derived the part of the polarized cross section containing the derivative term, with particular emphasis on the contribution caused by the color entanglement effect. This effect arises from the non-trivial interplay between one extra gluon exchange from the proton side and multiple gluon exchanges from the nucleus. The identified new gluon distribution $G_{4}\left(x_{g}^{\prime}, k_{\perp}\right)$ can be explicitly evaluated in the MV model. As a result, measuring this observable would provide us with a unique chance to quantitatively study color entanglement effects.

We have further shown that the spin dependent cross section computed in the standard collinear approach can be recovered from the hybrid approach in the kinematical region where the transverse momentum of the produced photon is much larger than the saturation momentum, provided that the contribution arises from color entanglement effect is not included in our result. However, at low photon transverse momentum, in sharp contrast to the predication from the standard collinear twist-3 approach, the spin asymmetry is found to be proportional to the photon transverse momentum.

A direct extension of this work is to investigate the impact of the color entanglement effect on the SSA in Drell-Yan lepton pair production in $\mathrm{p}^{\uparrow} \mathrm{A}$ collisions. One can also use the hybrid formalism to calculate the SSAs for pion production and di-jet and photon-jet 42 production in $\mathrm{p}^{\uparrow} \mathrm{A}$ collisions. A proposed $\mathrm{p}^{\uparrow} \mathrm{A}$ program at RHIC [4] is thus extremely welcome. Finally, we would like to emphasize that the hybrid approach in principal can also be applied to $\mathrm{p}^{\uparrow} \mathrm{p}$ collisions, where it, however, only

is valid in the very forward region at low transverse momentum $\Lambda_{Q C D} \ll l_{\gamma \perp} \leq Q_{s q}^{p}$ (where $Q_{s q}^{p}$ is the proton saturation momentum). In other words, the standard collinear twist-3 approach is not adequate to describe SSA phenomenology in $\mathrm{p}^{\uparrow} \mathrm{p}$ or $\mathrm{p}^{\uparrow} \mathrm{A}$ collisions in the mentioned kinematic region where the CGC framework can apply on target nucleus or proton side.

Acknowledgments: We are grateful to Bowen Xiao and Feng Yuan for reminding us of the tadpole type contribution to the gluon distribution $G_{4}$ in the MV model. This work has been supported by BMBF (OR 06RY9191).

\section{References}

[1] G. L. Kane, J. Pumplin and W. Repko, Phys. Rev. Lett. 41, 1689 (1978). 
[2] J. P. Ma and H. Z. Sang, JHEP 0811, 090 (2008).

[3] D. W. Sivers, Phys. Rev. D 43, 261 (1991).

[4] J. C. Collins, Nucl. Phys. B 396, 161 (1993).

[5] A. V. Efremov and O. V. Teryaev, Sov. J. Nucl. Phys. 36, 140 (1982) [Yad. Fiz. 36, 242 (1982)]; Phys. Lett. B 150, 383 (1985).

[6] J.-w. Qiu and G. F. Sterman, Phys. Rev. Lett. 67, 2264 (1991);

[7] J.-w. Qiu and G. F. Sterman, Nucl. Phys. B 378, 52 (1992).

[8] X. -D. Ji, Phys. Lett. B 289, 137 (1992).

[9] H. Beppu, Y. Koike, K. Tanaka and S. Yoshida, Phys. Rev. D 82, 054005 (2010).

[10] F. Yuan and J. Zhou, Phys. Rev. Lett. 103, 052001 (2009) arXiv:0903.4680 [hep-ph]]. Z. -B. Kang, F. Yuan and J. Zhou, Phys. Lett. B 691, 243 (2010) arXiv:1002.0399 [hep-ph]]. A. Metz and D. Pitonyak, arXiv:1212.5037 [hep-ph].

[11] D. Boer, P. J. Mulders and F. Pijlman, Nucl. Phys. B 667, 201 (2003) hep-ph/0303034.

[12] K. Kanazawa, Y. Koike, A. Metz and D. Pitonyak, arXiv:1404.1033 [hep-ph].

[13] Z. -B. Kang, J. -W. Qiu, W. Vogelsang and F. Yuan, Phys. Rev. D 83, 094001 (2011) arXiv:1103.1591 [hep-ph]].

[14] A. Metz, D. Pitonyak, A. Schäfer, M. Schlegel, W. Vogelsang and J. Zhou, Phys. Rev. D 86, 094039 (2012) arXiv:1209.3138 [hep-ph]].

[15] C. Kouvaris, J. -W. Qiu, W. Vogelsang and F. Yuan, Phys. Rev. D 74, 114013 (2006) hep-ph/0609238.

[16] L. Nogach [AnDY Collaboration], arXiv:1212.3437 [hep-ex]. L. C. Bland et al. [AnDY Collaboration], arXiv:1304.1454 [hep-ex].

[17] L. Gamberg and Z. -B. Kang, Phys. Lett. B 718, 181 (2012) arXiv:1208.1962 [hep-ph]].

[18] K. Kanazawa and Y. Koike, arXiv:1212.3071 [hep-ph].

[19] F. Yuan, Phys. Rev. D 78, 014024 (2008) arXiv:0801.4357 [hep-ph]].

[20] A. Schäfer and J. Zhou, Phys. Rev. D 88, no. 1, 014008 (2013) [arXiv:1302.4600 [hep-ph]].

[21] D. Boer, A. Dumitru and A. Hayashigaki, Phys. Rev. D 74, 074018 (2006).

[22] L. D. McLerran and R. Venugopalan, Phys. Rev. D 49, 2233 (1994); Phys. Rev. D 49, 3352 (1994).

[23] Z. -B. Kang and B. -W. Xiao, Phys. Rev. D 87, 034038 (2013) arXiv:1212.4809 [hep-ph]].

[24] Z. -B. Kang and F. Yuan, Phys. Rev. D 84, 034019 (2011).

[25] Y. V. Kovchegov and M. D. Sievert, Phys. Rev. D 86, 034028 (2012) [Erratum-ibid. D 86, 079906 (2012)]. 
[26] T. C. Rogers and P. J. Mulders, Phys. Rev. D 81, 094006 (2010) [arXiv:1001.2977 [hep-ph]].

[27] M. G. A. Buffing and P. J. Mulders, arXiv:1309.4681 [hep-ph].

[28] J. Bartels, B. I. Ermolaev and M. G. Ryskin, Z. Phys. C 70, 273 (1996); Z. Phys. C 72, 627 (1996).

[29] A. Metz and J. Zhou, Phys. Rev. D 84, 051503 (2011). A. Schäfer and J. Zhou, arXiv:1305.5042 [hep-ph].

[30] F. Dominguez, J. -W. Qiu, B. -W. Xiao and F. Yuan, Phys. Rev. D 85, 045003 (2012) arXiv:1109.6293 [hep-ph]].

[31] A. Schäfer and J. Zhou, Phys. Rev. D 85, 114004 (2012) arXiv:1203.1534 [hep-ph]]. T. Liou, Nucl. Phys. A 897, 122 (2013) arXiv:1206.6123 [hep-ph]].

[32] E. Akcakaya, A. Schäfer and J. Zhou, Phys. Rev. D 87, no. 5, 054010 (2013) arXiv:1208.4965 [hep-ph]].

[33] A. Schäfer and J. Zhou, arXiv:1308.4961 [hep-ph].

[34] J. Zhou, arXiv:1308.5912 [hep-ph].

[35] Y. V. Kovchegov and M. D. Sievert, arXiv:1310.5028 [hep-ph].

[36] T. Altinoluk, Ns. Armesto, G. Beuf, M. Martnez and C. A. Salgado, arXiv:1404.2219 [hep-ph].

[37] F. Gelis and J. Jalilian-Marian, Phys. Rev. D 66, 014021 (2002) hep-ph/0205037].

[38] I. Balitsky, Nucl. Phys. B463, 99-160 (1996). [hep-ph/9509348].

[39] L. D. McLerran, R. Venugopalan, Phys. Rev. D59, 094002 (1999). hep-ph/9809427].

[40] J. P. Blaizot, F. Gelis and R. Venugopalan, Nucl. Phys. A 743, 13 (2004) hep-ph/0402256].

[41] J. P. Blaizot, F. Gelis and R. Venugopalan, Nucl. Phys. A 743, 57 (2004) hep-ph/0402257].

[42] A. Schäfer and J. Zhou, arXiv:1406.3198 [hep-ph].

[43] E. C. Aschenauer, A. Bazilevsky, K. Boyle, K. O. Eyser, R. Fatemi, C. Gagliardi, M. GrossePerdekamp and J. Lajoie et al., arXiv:1304.0079 [nucl-ex]. 